$N$ additional patterns with their $N-1$ cyclic shifts. Thus there are

$$
\left[\left(2^{N}-2\right) / N\right]+2
$$

fundamental patterns that, together with all of their shifts, make up the $2^{N}$ patterns. (If one trains such a neural network to have $-1-1-1 \quad 1$ as a stable state, for example, then this pattern and its four shifts will all be stable states.)

This model uses the characteristic equation

$$
\begin{aligned}
V(i)= & \operatorname{sgn}\left\{\sum_{j} T(j) V((j+i))_{N}\right. \\
& +\sum_{j} \sum_{k} T(j, k) V((j+i))_{N} V((k+i))_{N} \\
& +\sum_{j} \sum_{k} \sum_{i} T(j, k, l) V((j+i))_{N} V((k+i))_{N} \\
& \left.\times V((l+i))_{N}+\ldots\right\}
\end{aligned}
$$

where the $((j+i))_{N}$ signifies $(j+i)$ modulo $N$. In this model all connection matrices are independent of the neuron for which the output is being calculated. The next state of a particular neuron depends only on the state of the other $N-1$ neurons and the relative distance between the neurons. For example, using a one-dimensional connection matrix

$$
\begin{aligned}
V(2)= & \operatorname{sgn}\{T(1) V(3)+T(2) V(4)+\ldots+T(N-3) V(N-1) \\
& +T(N-2) V(0)+T(N-1) V(1)\}
\end{aligned}
$$

These equations can easily be extended to higher-dimensional connection matrices. To store $2^{N-1}$ patterns using the above model requires a connection matrix of dimension $N-1$ with only one nonzero weight. Two examples are given below.

Example 3: Let $N=3$ and use a two-dimensional connection matrix for calculation of the next state:

$$
\begin{array}{r}
V(i)=\operatorname{sgn}\left\{\sum_{j} \sum_{k} T(j, k) V((j+i))_{N} V((k+i))_{N}\right\} \\
\text { with } j \neq 0, k \neq 0, k>j
\end{array}
$$

The even parity patterns can be stored using $T(1,2)=-1$ and all other $T(i, j)=0$.

Example 4: Let $N=4$ and use a three-dimensional connection matrix for calculation of the next state:

$$
\begin{aligned}
V(i)=\operatorname{sgn}\{ & \left\{\sum_{j} \sum_{k} \sum_{l} T(j, k, l) V((j+i))_{N}\right. \\
& \left.\times V((k+i))_{N} V((l+i))_{N}\right\} \\
& \text { with } j \neq 0, k \neq 0, k>j, l \neq 0, l>k
\end{aligned}
$$

The even parity patterns can be stored using $T(1,2,3)=1$ and all other $T(i, j, k)=0$.

Conclusion: It has been shown that extending the Hopfield model to make use of higher-order terms increases to $2^{N-1}$ the maximum number of patterns that can be stored simultaneously. Also, a new neural network model has been briefly introduced that automatically stores all cyclic shifts of a pattern as it stores that pattern. This model is also shown to be limited to $2^{N-1}$ stored patterns.

\section{L. PRADOS}

29th February 1988

Department of Electrical \& Computer Engineering

Louisiana State University

Baton Rouge, LA 70803, USA

\section{References}

1 MCELIECE, R. J., POSNER, E. C., RODEMICH, E. R., and VENKATESH, S. S.: 'The capacity of the Hopfield associative memory', IEEE Trans., 1987, IT-33, pp. 461-482

ELECTRONICS LETTERS 14th April 1988 Vol. 24 No. 8
2 CHEN, H. H., LEE, Y. C., SUN, G. Z., and LEE, H. Y.: 'High order correlation model for associative memory'. Proc. AIP Conference on Neural Networks for Computing, Snowbird, 1986, pp. 86-99

3 SEJNOWSKI, T. J.: 'Higher-order Boltzman machines'. Proc. AIP Conference on Neural Networks for Computing, Snowbird, 1986, pp. $398-403$

\section{HIGH-SPEED FRONT-ILLUMINATED GalnAsP/InP pin PHOTODIODE}

\begin{abstract}
Indexing terms: Semiconductor devices and materials, Photodiodes, Semiconductor growth, Integrated optics

We have developed a high-speed front-illuminated GaInAsP/ InP pin photodiode for use at the optical wavelength of $1.3 \mu \mathrm{m}$. The device is grown on an $n^{+}-$InP substrate and uses polyimide both as a passivation layer and as a bonding pad holder to reduce parasitic capacitance. An optoelectronic sampling measurement of the impulse response shows a pulsewidth (FWHM) of $28 \mathrm{ps}$. A $3 \mathrm{~dB}$ bandwidth in excess of $18 \mathrm{GHz}$ has been achieved.
\end{abstract}

Most of the high-speed photodiodes reported to date for use in the $1.3-1.6 \mu \mathrm{m}$ band have utilised a structure requiring back illumination. ${ }^{1-3}$ However, a front-illuminated device is sometimes preferred, particularly for a planar integrated structure. In this letter we report on a high speed front-illuminated GaInAsP/InP pin photodiode. At a bias voltage of $-5 \mathrm{~V}$, a dark current of $200 \mathrm{pA}$, and an external quantum efficiency of $20 \%$ have been measured. A $3 \mathrm{~dB}$ bandwidth in excess of $18 \mathrm{GHz}$ and an impulse response with a full width at half maximum (FWHM) of $28 \mathrm{ps}$ were observed.

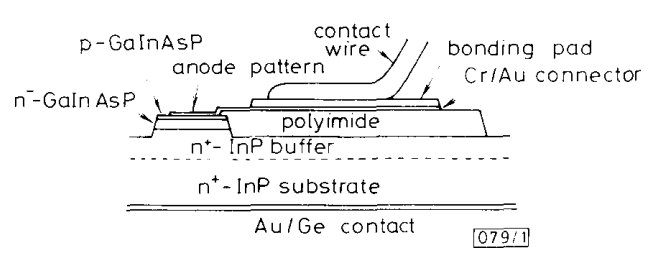

Fig. 1 Cross-sectional view of shallow diffusion photodiode

The diode structure is shown in Fig. 1 and the growth procedure is described in more detail elsewhere. ${ }^{4}$ An $n^{+}$-InP buffer layer and a $2 \mu \mathrm{m}$-thick $n^{-}-\mathrm{Ga}_{0.27} \mathrm{In}_{0.73} \mathrm{As}_{0.66} \mathrm{P}_{0.34}$ layer $\left(n \simeq 2 \times 10^{15} \mathrm{~cm}^{-3}, \lambda_{g} \simeq 1.37 \mu \mathrm{m}\right)$ are grown successively on an $n^{+}-$InP substrate by LPE. The $p n$-junction was then formed by shallow $C d$ diffusion into the $n^{-}-\operatorname{In}_{0.73} \mathrm{As}_{0.66} \mathrm{P}_{0.34}$ layer. $\mathrm{A} \mathrm{Cr} / \mathrm{Au}$ contact was then evaporated on the $p^{+}$GaInAsP in a finger pattern using a lift-off process. Next, chemical etching in $\mathrm{Br}$-methanol solution was used to define a mesa structure with an area of $25 \mu \mathrm{m} \times 30 \mu \mathrm{m}$ and a height of $2.5 \mu \mathrm{m}$. A $3 \mu \mathrm{m}$ layer of polyimide is then deposited. This is then patterned into $100 \mu \mathrm{m}$ square polyimide mesas which abutt the diode mesas by etching through a photoresist mask with the developer during an extended development time of the photoresist. The large tapered $\mathrm{Cr} / \mathrm{Au}$ connector layer and bonding pad were then successively patterned onto the polyimide/diode mesas.

The purpose of the polyimide layer is twofold: to passivate the diode $p n$-junction ${ }^{5}$ and to serve as an insulating layer which supports a large bonding pad for external access contact, resulting in a much reduced parasitic capacitance. In the present device, a circular bonding pad $50 \mu \mathrm{m}$ in diameter on a $3 \mu \mathrm{m}$-thick polyimide layer has a capacitance of less than $0.02 \mathrm{pF}$. No frequency dependence of the polyimide's dielectric constant was observed from $0-12 \mathrm{GHz}$, warranting a more complete and accurate measurement of the high-frequency properties.

The doping profile of the $p$-layer requires careful attention since the incident light must traverse it, creating carriers outside the depletion region. This can lead to adverse minority carrier diffusion effects. Three different $\mathrm{Cd}$ diffusion profiles 
in the GaInAsP, shown in Fig. 2, were measured with an ion microprobe. The three diffusions were done at a temperature of $600^{\circ} \mathrm{C}$, varying only the diffusion times which were 10,20 and $30 \mathrm{~min}$. The $10 \mathrm{~min}$ diffusion yielded a $0.7 \mu \mathrm{m}$ junction depth, and a surface concentration of $2 \times 10^{19} \mathrm{~cm}^{-3}$ which is almost the same as the $30 \mathrm{~min}$ diffusion indicating saturation. Using an exponential approximation for the diffusion profile, the transit time of minority electrons in the $p$ layer, $\tau_{\text {diff }}$, is estimated $^{6}$ to be $\tau_{\text {diff }} \simeq 3 \mathrm{ps}$.

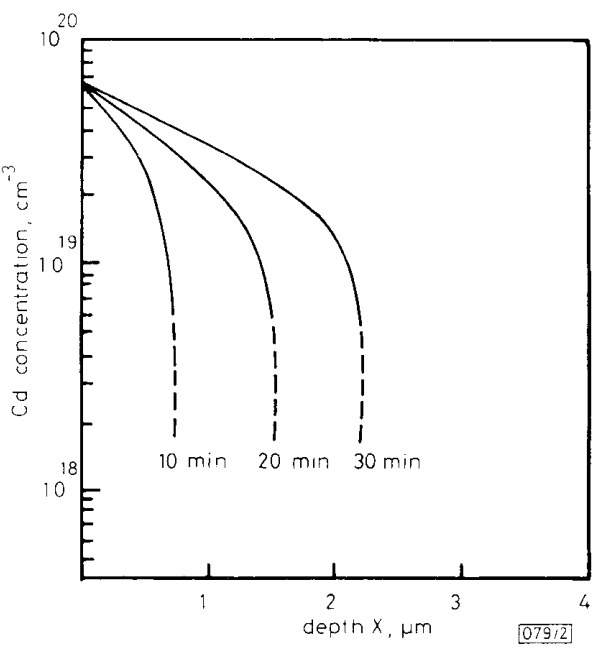

Fig. $2 \mathrm{Cd}$ diffusion profiles for diffusion times of 10,20 and $30 \mathrm{~min}$ as measured with an ion microprobe

Broken lines indicate limited accuracy region of measurement $\mathrm{Cd}$ diffusion in undoped $\left(n \equiv 3 \times 10^{5} \mathrm{~cm}^{-3}\right) \quad$ GaInAsP at $T=600^{\circ} \mathrm{C}$

The impulse response of the photodiode with a $0.7 \mu \mathrm{m}$ junction depth was tested by illuminating it with 4 ps light pulses from a mode-locked dye laser $(\lambda=6000 \AA)$. A Tektronix S-4 sampling head which had a rise time of 25 ps was employed for this measurement. The impulse response of the photodiode is 40 ps (FWHM) when operated at zero bias. This indicates that the shallow diffusion photodiode can be used as a high speed device at very low bias. The breakdown voltage of the diode was in excess of $50 \mathrm{~V}$. It was, however, typically operated at a reverse bias of $5 \mathrm{~V}$. At this bias an impulse response of 35 ps FWHM was observed, which is largely limited by the response of the sampling oscilloscope. Diodes made with a 30 minute $\mathrm{Cd}$ diffusion exhibited an expected long diffusion tail in the impulse response which was not observed with the shallow $p$-layer diode.

To better resolve the actual impulse response of the photodiode an optoelectronic sampling measurement was made by using a microstrip transmission-line structure on a proton bombarded semi-insulating GaAs substrate. ${ }^{7}$ The sampling gate time aperture was estimated to be $12 \mathrm{ps}$ from an autocorrelation measurement of the sampling geometry used. A

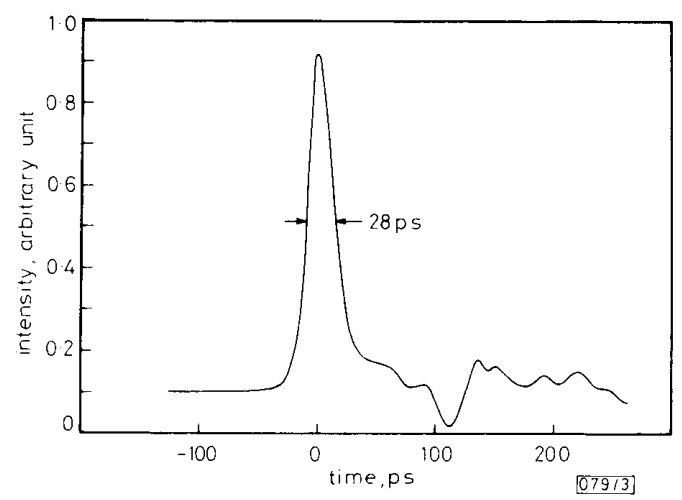

Fig. 3 Impulse response of photodiode measured by optoelectronic sampling technique typical sampling measurement is shown in Fig. 3 with a risetime of $12 \mathrm{ps}$ and a FWHM of $28 \mathrm{ps}$.

The high-speed performance of this photodiode was also evaluated by measuring its response in the frequency domain. An HP 8565A spectrum analyser was used to measure the output of the photodiode (Fig. 4) while illuminated by the same optical pulse source as used for the impulse response measurement. The rolloff may be at least partially due to instrument limitations rather than the ultimate photodiode response; nevertheless, the response is flat (within $3 \mathrm{~dB}$ ) to beyond $18 \mathrm{GHz}$. For comparison, the frequency response obtained by a fast Fourier transform (FFT) of the temporal impulse response is also shown in Fig. 4. The discrepancy between the two curves is probably due to the correction technique.

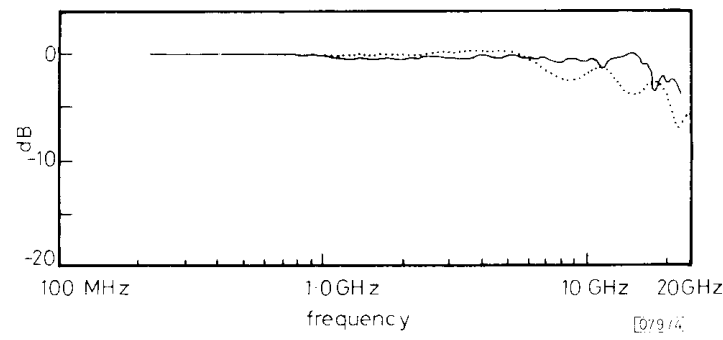

Fig. 4 Measured frequency responses of photodiode

- HP 8565A spectrum analyser

..... FFT of temporal impulse response with a correction for finite sampling window

Under pulsed excitation a responsivity of $0.1 \mathrm{~A} / \mathrm{W}$ which corresponds to an external quantum efficiency of $20 \%$ was measured. To reduce the effects of the sheet resistance and surface recombination of the Cd diffused $p$-layer, we intentionally left a semitransparent $\mathrm{Cr}$ film on the window area, and found a considerable increase in the sensitivity of the photodiode.

In conclusion we have fabricated a high-speed GaInAsP pin photodiode with a polyimide bonding pad holder on an $n^{+}-\operatorname{InP}$ substrate for use at the wavelength of $1.3 \mu \mathrm{m}$. The built-in field aided transport of electrons in the shallow diffused $p$-region has reduced the transit time of minority carriers in this front illuminated photodiode. A risetime of $12 \mathrm{ps}$ and a FWHM of $28 \mathrm{ps}$ have been achieved.

This work was supported by the National Science Foundation, the Office of Naval Research and the Air Force Office of Scientific Research. The authors wish to thank Bill Hamilton of the Jet Propulsion Laboratory for making the ion microprobe measurements of the Cd diffusion profiles.

M. B. YI*

24th February 1988

J. PASLASK

Y.-Y. LIU

T. R. CHEN'

A. YARIV

California Institute of Technology

Pasadena, CA 91125, USA

* On leave from Jilin University, Changchun, Jilin, People's Republic of China

$\dagger$ On leave from Chengdu Institute of Radio Engineering, Chengdu, Sichuan, People's Republic of China

\section{References}

1 LEE, T. P., BURRUS, C. A., OGaWA, K., and dental, A. G.: 'Very-high speed back-illuminated GaInAs/InP punch-through photodiodes', Electron. Lett., 1981, 17, pp. 431-432

2 LI, K., REZEK, E, and LAW, H. D.: 'InGaAs pin photodiode fabricated on semi-insulating InP substrate for monolithic integration', Electron. Lett., 1984, 20, p. 196

3 bowers, J. E., burrus, C. A., and tucker, R. S.: Conf. Picosecond Electronics and Optoelectronics Technical Digest, Incline Village, Nevada, March 13-15, 1985, paper ThA3-1

4 YI, M. B., PASlaSki, J., LU, L. T., MARGalit, S., YARIV, A., BLAUVELT, H., and LAU, K.: J. Appl. Phys., 1985, 58, p. 4730

5 YEATS, R., and VON DESSONNECK, K.: Appl. Phys. Lett., 1984, 44, p. 145

6 LUCOVSKY, G., and EMmons, R. B.: Appl. Optics, 1985, 4, p. 697

7 Auston, D. H., and smith, P. R.: Appl. Phys. Lett., 1981, 41, p. 599

ELECTRONICS LETTERS 14th April 1988 Vol. 24 No. 8 